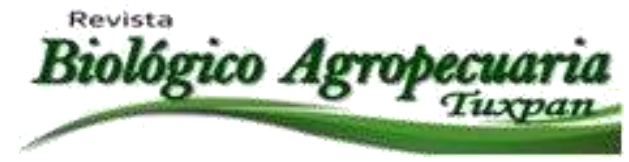

\title{
Extracciones nutrimentales en tres cultivos básicos en Veracruz, México
}

Nutrimental extractions in three basic crops in Veracruz, Mexico

Vásquez Hernández Andrés ${ }^{\bowtie}$, Rigoberto Zetina Lezama e Isaac Meneses Márquez

Instituto Nacional de Investigaciones Forestales, Agrícolas y Pecuarias. Centro de Investigación Regional del Golfo Centro. Campo Experimental Cotaxtla km 34 Carr. libre Veracruz-Córdoba. Apdo. Postal 429, C.P. 91700.tel y fax: 01(285) 59601 06, 07, 08,09.

${ }^{\square}$ Autor para correspondencia: vasquez.andres@inifap.gob.mx

Recibido: 09/01/2014

Aceptado: 13/07/2014

\section{RESUMEN}

El uso de los análisis de suelo como herramienta para hacer más eficiente el uso de los fertilizantes químicos aplicados, es de uso indispensable, ya que nos permite calcular de manera más confiable la cantidad de fertilizante a aplicar de manera sitio-especifica. Una alternativa para recomendar fertilización sitio especifica es el Método Racional de Fertilización, el cual es un cálculo de los requerimientos de fertilización basado en el análisis químico del suelo, en la extracción unitaria de nutrientes del cultivo y en la eficiencia de los fertilizantes. La tasa de extracción de nutrientes o extracción unitaria expresada en $\mathrm{kg}$ de nutriente por tonelada de grano cosechado debe ser determinada por cultivo y región, ya que nos indica de manera directa la cantidad de nutriente necesaria para obtener el máximo rendimiento potencial del cultivo. En este trabajo se determinó la extracción unitaria de nutrientes en los cultivos básicos maíz y frijol; también se incluyó caña de azúcar, por su gran importancia para el estado de Veracruz, donde se ubica la mayor cantidad de ingenios azucareros a nivel nacional. En frijol se tuvieron extracciones promedio de $41.5 \mathrm{~kg}$ de N, 6 de P y 40 de K. Para maíz la extracción fue de $27.3 \mathrm{~kg}$ de N, 5.9 de P y 29.1 de K. Par el caso de caña se tuvo una extracción de $1.43 \mathrm{~kg}$ de N, 0.30 de $\mathrm{P}$ y 1.80 de K.

Palabras clave: maíz, frijol, caña, extracciones unitarias.

\begin{abstract}
The use of the soil analysis as a tool to make more efficient use of chemical fertilizers applied, is essential to use, since it allows us to estimate more reliably the amount of fertilizer to apply site specific. An alternative to recommend fertilization site specified is the rational method of fertilization,
\end{abstract}

\section{Revista Científica Biológico Agropecuaria Tuxpan 2 (1)} ISSN: 2007-6940 
which is a calculation of the fertilization requirements based on the chemical analysis of soil, unitary extraction of crop nutrients and fertilizer efficiency. The rate of extraction of nutrients or extraction unit expressed in $\mathrm{kg}$ of nutrient per ton of harvested grain should be determined by culture and region, since it indicates directly the amount of nutrient needed for peak performance potential of the crop. In this work determined unitary extraction of nutrients in basic crops corn and beans; also included sugar cane by its great importance for the State of Veracruz, where is located the most sugar mills at the national level. Bean has extractions average of $41.5 \mathrm{~kg} \mathrm{~N}, 6 \mathrm{P}$ and $40 \mathrm{~K}$. For corn removal was $27.3 \mathrm{~kg}$ of N, 5.9 P and 29.1 K. For sugarcane the rule of extraction was $1.43 \mathrm{~kg}$ of N, $0.30 \mathrm{P}$ and $1.80 \mathrm{~K}$.

Keywords: corn, beans, sugarcane, unit extractions.

\section{INTRODUCCIÓN}

La fertilización de los cultivos es una actividad que por su costo es uno de los aspectos más importantes a considerar para obtener una producción aceptable desde el punto de vista económico. Las últimas predicciones de FAO (Food and Agriculture Organization) indican que para el año 2050 la población mundial será de 9,100 millones de habitantes, frente a los 6,800 millones actuales, esto representa un incremento del $34 \%$ para los próximos 40 años, con un consumo global de cereales per cápita de unos $340 \mathrm{~kg}$ por persona y año con un total de 3,094 millones de toneladas por año; en este aspecto, GarcíaSerrano et al. (2010) mencionan que es necesario mantener e incrementar los rendimientos de los cultivos, empleando técnicas que permitan practicar una agricultura productiva, pero también sostenible, en la que los fertilizantes se empleen de forma racional, con máxima eficiencia y respeto al medio ambiente.

El uso de los análisis de suelo como herramienta para hacer más eficiente el uso de los fertilizantes químicos, es indispensable, ya que nos permite calcular de manera confiable la cantidad de fertilizante a aplicar de manera sitio-especifica. Aunque hace algunos años la cantidad de fertilizante a aplicar se determinaba mediante el establecimiento de experimentos de dosis de fertilización en los ambientes más representativos para los cultivos de interés, la variabilidad que existe de un terreno o finca de cultivo a otra es muy grande, por lo que se pueden cometer errores apreciables con pérdida de precisión en la recomendación de fertilización. Una alternativa para recomendar fertilización sitio especifica es el Método Racional de Fertilización el cual es un método de cálculo de los requerimientos de fertilización basado en el análisis químico del suelo para determinar su disponibilidad de nutrientes esenciales, en la tasa de extracción unitaria de nutrientes determinada para cada cultivo en particular y en la eficiencia de los fertilizantes, la cual depende en gran medida de la disponibilidad de agua para las plantas(Bouz, Astegiano y Favaro, 2003) . La base técnica para implementar este método es la tasa de extracción unitaria de nutrientes, que es la cantidad de nutriente que el cultivo requiere por tonelada del producto a cosechar (Castellanos et al. 2005), y debe ser determinada por cultivo y región.

El planteamiento general proviene del método racional del Rodríguez (1993) quien propone la dosis de abonado del nutriente (Di) como resultante de la demanda del cultivo

Revista Científica Biológico Agropecuaria Tuxpan 2 (1) 
(DEMi), el suministro del suelo (SUMINi) y la eficiencia de la fertilización (EFi):

Dosis de fertilizante $=$ Demanda del cultivoSuministro del Suelo

\section{Eficiencia de la fertilización}

La eficiencia (E) de utilización del nutrimento se refiere a la cantidad del nutrimento recobrado y depende de: las propiedades del nutrimento, características del suelo, características de la fuente del fertilizante, método y época de aplicación, condiciones climáticas. Para el nitrógeno los valores fluctúan entre 40 y $60 \%$ utilizando valores más altos cuando las condiciones son menos propicias a pérdidas y más bajos cuando las condiciones del suelo o manejo son pobres. El rango práctico para estimar la eficiencia de la fertilización de P fluctúa entre 30 y $40 \%$ dependiendo de las condiciones y el tipo del suelo; para potasio se reportan valores de eficiencia de entre 50 y $60 \%$. En este estudio se determinó la tasa de extracción de nutrientes en cultivos básicos de gran importancia en la alimentación para el pueblo mexicano como son maíz y frijol; también se realizó el estudio para caña de azúcar, la cual es un cultivo industrial de gran importancia para el estado de Veracruz, en la que se encuentran ubicados la mayor cantidad de ingenios azucareros a nivel nacional.

\section{MATERIALES Y MÉTODOS}

Para maíz y frijol se realizó el estudio en la parte central de Veracruz, en los municipios de Jamapa, Cotaxtla y Medellín de Bravo, región en la que tradicionalmente se han sembrado estos cultivos; para caña de azúcar se estudiaron los municipios de
Actopan, Ursulo, Galván y Paso de Ovejas en Veracruz , también se incluyó el municipio de Acatlán, Oaxaca por su colindancia con Veracruz, dado que gran parte de esta producción también se procesa en el estado de Veracruz $o$ en ingenios colindantes. Se seleccionaron 5 fincas de frijol, 5 de maíz y 11 de caña de azúcar en las regiones de estudio mencionadas. A la madurez fisiológica de los cultivos se realizó un muestreo para estimar el rendimiento y biomasa aérea de tallos y hojas. Para frijol y maíz, se muestrearon 4 surcos de 10 metros lineales con 4 repeticiones, haciendo una separación de grano, tallos y hojas, tomando muestras de cada parte para la determinación de humedad y hacer la corrección a base seca; para el caso de hojas y tallos de caña, se obtuvo una muestra representativa de cada repetición para hacer los análisis químicos de nutrientes. Para el análisis total de nutrientes, se usó la técnica de digestión húmeda (Jones, 1991) con

cuantificación de nutrientes por espectrofotometría de absorción atómica y fotocolorimetría (Plenecassagne, Romero y López, 1997). Con los datos de rendimiento de grano, tallo, hojas y la composición química de nutrimentos, se calculó la Extracción Unitaria de Nutrimentos por tonelada de grano para maíz y frijol y por tallos frescos para caña de azúcar.

\section{RESULTADOS Y DISCUSIÓN}

\section{Frijol}

Los rendimientos de frijol mostraron valores desde $752 \mathrm{~kg} \mathrm{ha}^{-1}$ hasta $2447 \mathrm{~kg} \mathrm{ha}^{-1}$ con un promedio de $1767 \mathrm{~kg} \mathrm{ha}^{-1}$, y un índice de cosecha promedio de 0.50 , los cuales son más altos que el promedio nacional de 646 $\mathrm{kg} / \mathrm{ha}$ (SIAP, 2005). En el cuadro 1 se muestran los resultados obtenidos para las extracciones unitarias en frijol, lo que de cierta manera nos

Revista Científica Biológico Agropecuaria Tuxpan 2 (1) ISSN: 2007-6940 
indica la cantidad mínima necesaria de nutrientes que se deben aportar al cultivo por cada tonelada de grano que se desee producir, sin obviar el potencial agroclimático de la región y del genotipo, así como la información obtenida en los experimentos para determinar la superficie de respuesta con dosis de fertilizantes. Para $\mathrm{N}$ se tuvo una extracción con una variación de 36.28 a 50.20, 4.86 a 7.71 para $\mathrm{P}$ y de 34.99 a $45.95 \mathrm{~kg} \mathrm{ha}^{-1}$ para $\mathrm{K}$, esto explica en cierta forma el hecho que el promedio de fertilización para frijol sea el tratamiento 40-40-00, de acuerdo a las recomendaciones del paquete tecnológico del INIFAP para frijol (1995). En base al cuadro 1 la extracción unitaria promedio de nutrientes para frijol quedo en el siguiente orden: $41.5 \mathrm{~N}>$ $40.0 \mathrm{~K}>14.8 \mathrm{Ca}>6 \mathrm{P}>5.3 \mathrm{Mg}>0.75 \mathrm{~S}>0.06$ $\mathrm{B}$, lo cual es el requerimiento mínimo de nutrientes necesarios para producir 1 ton de grano de frijol y nos servirá de base para calcular la fertilización para el cultivo mediante el Método Racional.

Cuadro 1. Rendimiento de grano, materia seca (M.S) de rastrojo y extracción unitaria de nutrientes en frijol negro.

\begin{tabular}{|c|c|c|c|c|c|c|c|c|c|c|c|}
\hline \multirow{2}{*}{ Localidad } & \multicolumn{3}{|c|}{ Kg ha $^{-1}$} & \multicolumn{8}{|c|}{ Extracción $\mathrm{Kg}$ ton- ${ }^{1}$ grano } \\
\hline & $\begin{array}{l}\text { M.S. } \\
\text { Grano }\end{array}$ & $\begin{array}{c}\text { Rastr } \\
\text { ojo }\end{array}$ & G + R & I.C. & N. & $\mathbf{P}$ & $\mathbf{K}$ & $\mathbf{C a}$ & Mg & $\mathbf{S}$ & B \\
\hline La Venta, M.F.A. & 752 & 1015 & 1767 & 0.43 & 36.3 & 4.9 & $\begin{array}{l}43 . \\
6\end{array}$ & $\begin{array}{l}12 . \\
2\end{array}$ & 5.8 & $\begin{array}{l}0.5 \\
9\end{array}$ & $\begin{array}{l}0.0 \\
5\end{array}$ \\
\hline $\begin{array}{l}\text { Espinal, Manlio } \\
\text { F.A. }\end{array}$ & 1646 & 1653 & 3299 & 0.50 & 40.5 & 6.7 & $\begin{array}{l}37 . \\
9\end{array}$ & $\begin{array}{l}17 . \\
5\end{array}$ & 5.9 & $\begin{array}{l}0.6 \\
5\end{array}$ & $\begin{array}{l}0.0 \\
5\end{array}$ \\
\hline Javilla, Jamapa & 2447 & 1703 & 4150 & 0.59 & 41.4 & 5.4 & $\begin{array}{l}37 . \\
7\end{array}$ & 7.0 & 3.9 & $\begin{array}{l}0.4 \\
1\end{array}$ & $\begin{array}{l}0.0 \\
5\end{array}$ \\
\hline $\begin{array}{l}\text { La Brecha, } \\
\text { Jamapa }\end{array}$ & 1615 & 1976 & 3591 & 0.45 & 50.2 & 7.7 & $\begin{array}{l}46 . \\
0\end{array}$ & $\begin{array}{l}23 . \\
4\end{array}$ & 6.5 & $\begin{array}{l}0.7 \\
5\end{array}$ & $\begin{array}{l}0.0 \\
8\end{array}$ \\
\hline $\begin{array}{l}\text { Las Minas, } \\
\text { Cotaxtla }\end{array}$ & 2377 & 2160 & 4537 & 0.53 & 39.4 & 5.5 & $\begin{array}{l}35 . \\
0\end{array}$ & $\begin{array}{l}13 . \\
8\end{array}$ & 4.3 & $\begin{array}{l}1.3 \\
5\end{array}$ & $\begin{array}{l}0.0 \\
6\end{array}$ \\
\hline Media & 1767 & 1701 & 3649 & $\begin{array}{l}0.50 \\
0\end{array}$ & 41.5 & 6.0 & $\begin{array}{l}40 . \\
0\end{array}$ & $\begin{array}{l}14 . \\
8\end{array}$ & 5.3 & $\begin{array}{l}0.7 \\
5\end{array}$ & $\begin{array}{l}0.0 \\
6\end{array}$ \\
\hline Mínimo & 752 & 1015 & 1767 & $\begin{array}{l}0.43 \\
0\end{array}$ & 36.3 & 4.9 & $\begin{array}{l}35 . \\
0\end{array}$ & 7.0 & 3.9 & $\begin{array}{l}0.4 \\
1\end{array}$ & $\begin{array}{l}0.0 \\
5\end{array}$ \\
\hline Máximo & 2447 & 2160 & 4537 & $\begin{array}{l}0.59 \\
0\end{array}$ & 50.2 & 7.7 & $\begin{array}{l}45 . \\
9\end{array}$ & $\begin{array}{l}23 . \\
4\end{array}$ & 6.5 & $\begin{array}{l}1.3 \\
5\end{array}$ & $\begin{array}{l}0.0 \\
8\end{array}$ \\
\hline $\begin{array}{l}\text { Desviación } \\
\text { estándar. }\end{array}$ & 690 & 436 & 1066 & $\begin{array}{l}0.06 \\
4\end{array}$ & 5.2 & 1.2 & 4.6 & 6.1 & 1.12 & $\begin{array}{l}0.3 \\
6\end{array}$ & $\begin{array}{l}0.0 \\
2\end{array}$ \\
\hline
\end{tabular}

Maíz

Revista Científica Biológico Agropecuaria Tuxpan 2 (1) 
En cuanto a maíz, los rendimientos de grano encontrados varían de 3711 a $6375 \mathrm{~kg}$ $\mathrm{ha}^{-1}$ con un índice de cosecha promedio de 0.36; el rendimiento de grano fue más alto que el promedio nacional de $1.595 \mathrm{~kg} \mathrm{ha}^{-1}$ de grano. En cuanto a la extracción de nutrimentos (Cuadro 2), se encontró un valor promedio de $27.3 \mathrm{~kg} \mathrm{~N}$ ton $^{-1}$ de grano, aunque todos los sitios fueron ligeramente más altos que los reportados en la literatura de $22.5 \mathrm{~kg} \mathrm{ton}^{-1} \mathrm{de}$ grano (Castellanos et al, 2006); fósforo varió de 4.3-8.3 kg ton ${ }^{-1}$ de grano, también mostrando valores superiores al promedio internacional de $4 \mathrm{~kg}$ ton $^{-1}$ de grano; potasio también mostro valores superiores al promedio internacional de $19 \mathrm{~kg}$ ton $^{-1}$. Calcio y Magnesio son semejantes a los valores internacionales, pero azufre está bajo.

Cuadro 2. Rendimiento de rastrojo, grano y extracciones unitarias en maíz en Veracruz.

\begin{tabular}{lcccccccccc}
\hline & M.S. & $\begin{array}{c}\text { Kg ha- }^{1} \\
\text { L.S. }\end{array}$ & Total & & & & & & & \\
\multicolumn{1}{c}{ Localidad } & & & & I.C. & N & P & K & Ca & Mg & S \\
& Rastrojo & Grano & $\mathbf{R}+\mathbf{G}$ & & & & & & & \\
\hline C. Ejidal, Cotaxtla & 10521 & 4828 & 15349 & 0.32 & 25.7 & 5.9 & 19.9 & 4.5 & 3.1 & 1.8 \\
Espinal, M.F.A. & 13629 & 6368 & 19997 & 0.32 & 25.1 & 4.3 & 29.0 & 2.5 & 2.2 & 1.2 \\
Cotaxtla & 8332 & 6375 & 14707 & 0.44 & 26.8 & 5.9 & 33.5 & 2.1 & 1.9 & 1.4 \\
Dos Lomas, Cotaxtla & 11212 & 5056 & 16268 & 0.31 & 31.3 & 8.3 & 43.2 & 3.2 & 3.2 & 1.4 \\
Remolino, & 5340 & 3711 & 9051 & 0.41 & 27.8 & 5.1 & 19.7 & 6.3 & 2.1 & 1.3 \\
Tlalixcoyan & & & & & & & & & & \\
Media & 9807 & 5268 & 15074 & 0.36 & 27.3 & 5.9 & 29.1 & 3.7 & 2.5 & 1.4 \\
Mínimo & 5340 & 3711 & 9051 & 0.31 & 25.1 & 4.3 & 19.7 & 2.1 & 1.9 & 1.2 \\
Máximo & 13629 & 6375 & 19997 & 0.44 & 31.3 & 8.3 & 43.2 & 6.3 & 3.2 & 1.8 \\
Desviación estd. & 3131 & 1129 & 3942 & 0.06 & 2.44 & 1.49 & 9.89 & 1.7 & 0.6 & 0.23 \\
\hline
\end{tabular}

Tomando en cuenta los datos del cuadro 2 , se puede indicar que el orden de extracción de nutrientes en maíz es de la siguiente manera: $29.1 \mathrm{~K}>27.3 \mathrm{~N}>5.9 \mathrm{P}>3.7 \mathrm{Ca}>2.5 \mathrm{Mg}>1.4 \mathrm{~S}$.

\section{Caña de azúcar}

Los rendimientos de tallos frescos variaron de 34186 a 184 976, con una media de 98045 ton ha ${ }^{-1}$ observándose que hay localidades por debajo de la media nacional de 72.6 ton ha $^{-1}$ (SIAP, 2006); el índice de cosecha en base a peso fresco de hojas y tallos tuvo un valor promedio de 0.87 . En el cuadro 3 se muestran los datos obtenidos en cuanto a las extracciones unitarias calculadas; por cada tonelada de caña producida se requirió en promedio $1.43 \mathrm{~kg}$ de nitrógeno, $0.30 \mathrm{~kg}$ de $\mathrm{P}$, $1.80 \mathrm{~kg}$ de $\mathrm{K}, 0.39 \mathrm{~kg}$ de $\mathrm{Ca}, 0.19 \mathrm{~kg}$ de $\mathrm{Mg}$ y $0.18 \mathrm{~kg}$ de $\mathrm{S}$, todos estos valores se encuentran dentro del rango reportado en la literatura (Malavolta y col. 1997; Chávez, 1999); en cuanto a los micronutrientes $\mathrm{B}, \mathrm{Fe}, \mathrm{Cu}, \mathrm{Zn}$ y Mn también están dentro del rango reportado. $\mathrm{El}$ orden unitario de extracción de nutrientes en $\mathrm{kg}$ ton $^{-1}$ de tallos frescos es: $1.80 \mathrm{~K}>1.43$ $\mathrm{N}>0.39 \mathrm{Ca}>0.30 \mathrm{P}>0.19 \mathrm{Mg}>0.8 \mathrm{~S}$; para el caso de los micronutrientes en gramos ton ${ }^{-1}$ de tallos frescos, el orden es el siguiente: 26.05 $\mathrm{Fe}>10.93 \mathrm{Mn}>4.25 \mathrm{Zn}>3.93 \mathrm{Cu}>1.82 \mathrm{~B}$. 
Cuadro 3. Rendimiento de tallos y hojas frescas e Índice de cosecha en caña de azúcar.

\begin{tabular}{lrrrr}
\hline \multicolumn{1}{c}{ Localidad } & \multicolumn{3}{c}{ Peso en kg } & \\
\cline { 2 - 3 } & $\begin{array}{c}\text { Hojas } \\
\text { frescas. }\end{array}$ & $\begin{array}{c}\text { Tallos } \\
\text { frescos }\end{array}$ & $\begin{array}{c}\text { Peso total } \\
\text { Biomasa }\end{array}$ & Índice de cosecha \\
1. Mozomboa, Actopan, Veracruz & 12018 & 93263 & 105281 & 0.89 \\
2. Zempoala, Ursulo G. Veracruz & 22780 & 176789 & 199569 & 0.88 \\
3. El Porvenir, Paso de O. Veracruz & 14142 & 109746 & 123888 & 0.89 \\
4. La Conquista, Paso de Ovejas, & 12872 & 99900 & 112772 & 0.89 \\
$\quad$ Ver. & 8526 & 66158 & 74684 & 0.89 \\
5. La Mata, Paso de Ovejas, & & & & \\
$\quad$ Veracruz & 23836 & 184976 & 208812 & 0.89 \\
6. Salmoral, Paso de Ovejas, & & & & \\
$\quad$ Veracruz & 9950 & 77221 & 87171 & 0.89 \\
7. Vicente Camalote, Acatlán, & & & & \\
$\quad$ Oaxaca & 8846 & 68647 & 77493 & 0.89 \\
8. Cañamazal I, Acatlán, Oaxaca & 8504 & 34186 & 42690 & 0.80 \\
9. Cañamazal II, Acatlán, Oaxaca & 20032 & 80540 & 100572 & 0.80 \\
10. Acatlán I, Oaxaca & 21656 & 87067 & 108723 & 0.80 \\
11. Acatlán II, Oaxaca & 14833 & 98045 & 112878 & 0.87 \\
Media & 8504 & 34186 & 42690 & 0.80 \\
Mínimo & 23836 & 184976 & 208812 & 0.89 \\
Máximo & 6079 & 45542 & 50363 & 0.042 \\
Desviación estándar & & & & \\
\hline
\end{tabular}

Cuadro 4. Extracciones unitarias de macro y micronutrientes en caña de azúcar.

\begin{tabular}{|c|c|c|c|c|c|c|c|c|c|c|c|}
\hline \multirow[t]{2}{*}{ Localidad } & \multicolumn{6}{|c|}{$\mathrm{Kg} \mathrm{ton}^{-1}$ de tallos frescos } & \multicolumn{5}{|c|}{$\mathrm{g} \mathrm{ton}^{-1}$ de tallos frescos } \\
\hline & $\mathbf{N}$ & $\mathbf{P}$ & $\mathbf{K}$ & $\mathbf{C a}$ & Mg & $\mathbf{S}$ & B & Fe & $\mathbf{C u}$ & $\mathbf{Z n}$ & Mn \\
\hline 1. Mozomboa & 0,75 & 0.22 & 1.36 & 0.42 & 0.16 & 0.20 & 4.50 & 13.19 & 4.07 & 4.18 & $\overline{5.04}$ \\
\hline 2. Zempoala & 1,36 & 0.32 & 1.80 & 0.37 & 0.19 & 0.18 & 1.13 & 53.17 & 2.09 & 3.34 & 7.46 \\
\hline 3. El Porvenir & 1,55 & 0.25 & 1.52 & 0.30 & 0.17 & 0.21 & 2.97 & 20.41 & 1.82 & 3 & 5.83 \\
\hline 4. La Conquista & 1,07 & 0.53 & 2.09 & 0.21 & 0.15 & 0.21 & 2.73 & 20.08 & 2.7 & 4.3 & 11.5 \\
\hline 5. La Mata & 2,29 & 0.35 & 3.44 & 0.29 & 0.19 & 0.14 & 2.10 & 25.6 & 4.69 & 4.23 & 4.69 \\
\hline 6. Salmoral & 1,14 & 0.33 & 1.45 & 0.44 & 0.12 & 0.21 & 1.18 & 12.65 & 3.68 & 3.46 & 3.73 \\
\hline 7. Vicente C. & 1,44 & 0.32 & 1.24 & 0.37 & 0.21 & 0.23 & 1.32 & 24.95 & 3.63 & 2.85 & 8.42 \\
\hline 8. Cañamazal & 1,29 & 0.16 & 1.58 & 0.22 & 0.07 & 0.07 & 0.50 & 15.56 & 4.37 & 3.64 & 24.61 \\
\hline 9. Cañamazal & 1,76 & 0.34 & 2.12 & 0.60 & 0.26 & 0.23 & 2.22 & 38.09 & 5.85 & 6.14 & 16.09 \\
\hline 10. Acatlán I & 1,49 & 0.21 & 2.03 & 0.59 & 0.26 & 0.11 & 1.20 & 26.82 & 5.34 & 6.33 & 21.6 \\
\hline 11. Acatlán II & 1,56 & 0.29 & 1.15 & 0.53 & 0.30 & 0.15 & 0.15 & 36.06 & 4.94 & 5.28 & 11.26 \\
\hline
\end{tabular}

Revista Científica Biológico Agropecuaria Tuxpan 2 (1) 
Vásquez et al., 2014

\begin{tabular}{lllllllllllr}
\hline Media & 1,43 & 0.30 & 1.80 & 0.39 & 0.19 & 0.18 & 1.82 & 26.05 & 3.93 & 4.25 & 10.93 \\
Mínimo & 0,75 & 0.16 & 1.15 & 0.21 & 0.07 & 0.07 & 0.15 & 12.65 & 1.82 & 2.85 & 3.73 \\
Máximo & 2,29 & 0.53 & 3.44 & 0.60 & 0.30 & 0.23 & 4.50 & 53.17 & 5.85 & 6.33 & 24.6 \\
$\begin{array}{l}\text { Desviación } \\
\text { Estándar }\end{array}$ & 0,38 & 0.09 & 0.61 & 0.13 & 0.06 & 0.05 & 1.19 & 11.70 & 1.24 & 1.14 & 6.73 \\
\hline
\end{tabular}

\section{CONCLUSIONES}

Frijol negro mostro tasa unitarias de extracción de $41.5 \mathrm{~kg}$ de $\mathrm{N}$ ton ${ }^{-1}$ de grano, 6 de $\mathrm{P}$ y 40 de $\mathrm{K}$, con un orden en la tasa unitaria de extracción: $41.5 \mathrm{~N}>40.0 \mathrm{~K}>14.8 \mathrm{Ca}>6 \mathrm{P}$ $>5.3 \mathrm{Mg}>0.75 \mathrm{~S}>0.06 \mathrm{~B}$. 2. El cultivo de maíz mostro tasas unitarias de extracción de $27.3 \mathrm{~kg} \mathrm{de} \mathrm{N} \mathrm{t}^{-1}$ de grano, 5.9 de $\mathrm{P}$ y 29.1 de $\mathrm{K}$, con un orden en la tasa unitaria de extracción: $29.1 \mathrm{~K}>27.3 \mathrm{~N}>5.9 \mathrm{P}>3.7 \mathrm{Ca}>2.5 \mathrm{Mg}>1.4 \mathrm{~S}$.

3. El cultivo de caña de azúcar mostró tasas unitarias de extracción de $1.43 \mathrm{~kg}$ de $\mathrm{N} \mathrm{t}^{-1}$ de tallos frescos, 0.30 de $\mathrm{P}$ y $1.80 \mathrm{de} \mathrm{K}$, El orden unitario de extracción de nutrientes en kg ton-1 de tallos frescos es: $1.80 \quad \mathrm{~K}>1.43 \quad \mathrm{~N}>0.39$ $\mathrm{Ca}>0.30 \mathrm{P}>0.19 \mathrm{Mg}>0.8 \mathrm{~S}$; para el caso de los micronutrientes en gramos ton $^{-1}$ de tallos frescos, el orden es el siguiente: 26.05 $\mathrm{Fe}>10.93 \mathrm{Mn}>4.25 \mathrm{Zn}>3.93 \mathrm{Cu}>1.82 \mathrm{~B}$.

\section{LITERATURA CITADA}

Agro-4037. 2001. Fertilidad de suelos y abonos. Recomendación de dosis de aplicación de nutrimentos. http://academic.uprm.edu/dsotomayor/a gro4037/handouts/4037_Handouts_V.pd f. Consultado el 20 de mayo de 2013.

Bouzo, C. A., Astegiano, E. D. y Favaro, J.C. 2003. Procedimiento para predecir la necesidad de abonos en cultivos hortícolas. Revista FAVE - Ciencias Agrarias 2 (1-2) 2003. 1-13 p. https://doi.org/10.14409/fa.v2i1/2.71
Castellanos, J. Z. 2005. La fertilización en los cultivos de maíz, sorgo y trigo en México. Folleto Técnico No. 1. INIFAP; CEB; SAGARPA. 44 p.

García-Serrano, J. P., Lucena M. J. J., Ruano C. S. y Nogales G. M. 2010. Guía Práctica de la Fertilización Racional de los Cultivos en España. Parte I. Gobierno de España. Ministerio del Medio Ambiente y Medio Rural y Marino. España. 120 p. https://doi.org/10.32418/ffs.2008.250.2089

INIFAP. 1995. Guía para la asistencia técnica agrícola en el área de influencia del Campo Experimental Cotaxtla. Instituto Nacional de Investigaciones Forestales, Agrícolas y Pecuarias. Centro de Investigación Regional del Golfo Centro. Campo Experimental Cotaxtla. Veracruz, Veracruz. México. $139 \mathrm{p}$. https://doi.org/10.20937/rica.2019.35.01.18

Rodríguez. 1993. La fertilización de los cultivos: Un método racional. Colección en Agricultura. Pontificia Universidad Católica de Chile. Chile. 291pp. https://doi.org/10.7764/cdi.38.964

SIAP-SAGARPA. 2005. Situación actual y perspectivas de la producción de frijol en México. 
Vásquez et al., 2014

Copyright (c) 2014 Andrés Vásquez Hernández, Rigoberto Zetina Lezama y Isaac Meneses Márquez

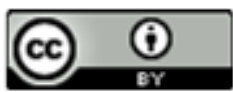

Este texto está protegido por una licencia Creative Commora 4.0 .

Usted es libre para Compartir —copiar y redistribuir el material en cualquier medio o formato-y Ad aptar el documento —remezclar, transformar y crear a partir del material- para cualquier propósito, inchso para fines comerciales, siempre que cumpla la condición de:

Atribución: Usted debe dar crédito a la obra original de manera adecuada, proporcionar un enlace a la licencia, e indicar si se han realizado cambios. Puede hacerlo en cualquier forma razonable, pero no de forma tal que sugiera que tiene el apoyo del licenciante o lo recibe por el usoque hace de la obra.

Resumendelicencia - Textocomplerodelalicenia 